\title{
Fulminant culture negative endophthalmitis as a new entity, toxic posterior segment syndrome?
}

\begin{abstract}
Purpose: To report an unusual case of sterile versus bacterial endophthalmitis in a post cataract surgery wound leak

Subject: A 58-year-old woman with a past eye medical history of LASIK underwent routine cataract extraction (CE) with lens implant of left eye and presented with a delayed, fulminant endophthalmitis OS on day 19 post CE or 3.5 days after the development of a post-op wound leak. Eye examination OS showed visual acuity (VA) of bare LP. Slit lamp examination revealed an edematous cornea with a $1 \mathrm{~mm}$ hypopyon. A large amount of exudate filled the entire posterior segment.

Results: Patient was treated with intravitreal vancomycin $(1 \mathrm{mg})$ and ceftazidime $(2 \mathrm{mg})$ and pars plana vitrectomy. Vitreal cultures were negative, but initial clinical presentation mimicked a virulent strep, staph, or pseudomonas endophthalmitis. Her postoperative course showed gradual improvement, reaching baseline VA of 20/30 OS with a normal eye exam.

Conclusion: Although culture-negative bacterial endophthalmitis is common, good visual outcomes with virulent bacteria is not. Fulminant culture-negative endophthalmitis with a good visual outcome may be due to a sterile toxic insult, a variation of TASS which we call Toxic Posterior Segment Syndrome (TPSS).
\end{abstract}

Keywords: endophthalmitis, culture-negative, toxic anterior segment syndrome, toxic posterior segment syndrome, postoperative wound leak, bare LP
Volume 4 Issue 6 - 2016

\author{
Stephen LoBue,' Thomas D LoBue \\ 'St Georges University School of Medicine, USA \\ ${ }^{2}$ LoBue Laser \& Eye Medical Centers, USA
}

Correspondence: Thomas D LoBue, Medical Director and CEO at LoBue Laser and Eye Medical Center Inc. 40700 California Oaks Rd, Ste 106, Murrieta, CA 92562, USA, Email Td1917@yahoo.com

Received:September 05, 2016 | Published: September 14, 2016
Abbreviations: CE, cataract extraction; VA, visual acuity; TASS, toxic anterior segment syndrome; TPSS, toxic posterior segment syndrome; BCVA, best corrected visual acuity; OD, right eye; OS, left eye

\section{Introduction}

Endophthalmitis in post-cataract surgeries is a rare complication occurring in $0.1 \%$ of cases in the United States. ${ }^{1,2}$ Bacterial infection is the most common agent in the US with coagulase-negative staphylococci being responsible for about $70 \%$ of post-cataract surgery endophthalmitis. Other typical bacteria include Staphylococcus aureus, beta and alpha hemolytic Streptococcus, and Pseudomonas aeruginosa ${ }^{3,4} \mathrm{~A}$ majority of symptoms occur around 1-2 weeks with the patients complaining of blurred vision, red eye, and eye pain. ${ }^{5}$

\section{Case report}

A 58-year-old woman was seen on 10/10/2014 complaining of blurry vision in both eyes that was getting progressively worse for the past year. The patient had difficulty reading and driving at night due to glare. The severity was said to be increasing as per the patient. Her past medical history was non-contributory. Past eye medical history was significant for bilateral myopic monovision LASIK in January 2001. On evaluation, her best corrected visual acuity (BCVA) was 20/40 in the right eye (OD) and 20/40-1 in the left eye (OS). Her intraocular pressures were $14 \mathrm{mmHg}$ OD and $12 \mathrm{mmHg}$ OS. Slit-lamp examination revealed $3+$ nuclear sclerotic cataracts bilaterally. The fundus was within normal limits. Before cataract surgery, the patient was referred to the retina service for examination of the peripheral retina due to a long axial length of $26 \mathrm{~mm}$. Fundus examination was unremarkable. The patient underwent uneventful cataract extraction OD on November 19, 2014, which resulted in excellent uncorrected near vision. Postoperative course was uneventful including suture removal at two weeks.

The patient underwent cataract extraction OS on 12/17/14. Her preoperative regimen included ketorolac and moxifloxacin 1 drop TiD for three days prior to surgery. On the day of surgery, the patient received drops of moxifloxacin, ketorolac, $1 \%$ cyclogyl, and $2.5 \%$ neosynephrine Q5min x 3. One drop of povidone-iodine 5\% was placed in the OS half an hour before surgery. The patient was brought into the OR and the ocular adnexa was cleansed with $5 \%$ povidoneiodine solution and the conjunctival fornices were irrigated with the same solution. Sterile drapes were applied and cataract extraction was performed under topical anesthesia using a temporal clear corneal tunnel incision with a disposable $2.4 \mathrm{~mm}$ metal blade. A foldable acrylic lens was placed in the capsular bag. The irrigation solution during surgery contained vancomycin. At the end of the case the wound was checked and found to be water tight. A single 10.0 nylon suture was placed with the knot buried. Even though the wound was water tight, suture placement was routinely done in patients who had previous LASIK surgery.

Postoperative day 1 the patient had no pain or visual complaints in the left eye and was seeing 20/30 uncorrected for distance. She was 
started on prednisolone, ketorolac, and moxifloxacin QID and was told to use an eye shield for sleeping. Her two week follow up was on $1 / 5 / 15$. She was without pain or visual complaints. Her uncorrected visual acuity was $20 / 30+$ OS. A drop of ciprofloxacin was placed OS and the corneal suture was removed under topical anesthesia at the slit lamp. By cutting the suture at its exit from the corneal stroma, care was taken not to drag exposed suture through the stroma. Postsuture removal, the wound was seidel negative and showed excellent integrity. The following day the patient stated she woke up with blurry vision. She was immediately seen in the office. The VA was counting fingers at 5 feet without improvement with pinhole. IOP was measured at $2 \mathrm{mmHg}$. Slit lamp exam showed that the incision was now seidel positive, with $2+$ corneal stromal folds, and a partially formed anterior chamber without cells, fibrin, or hypopyon. The posterior exam showed good red reflex and clear media. A bandaged contact lens was placed over the eye and moxifloxacin was increased to QiD. Prednisolone and Ketorolac were discontinued. The patient was scheduled for the OR the next day for suture placement OS.

The patient was taken to the OR as the first case at 0730 on 1/7/15. No eye prep was performed before surgery due to concern for a leaking wound. In the operating room, the bandage contact lens was removed and the wound was found to be seidel negative. The wound showed good integrity. Since the eye was intact and the incision was not open, a single 10.0 nylon suture was placed at a depth of midstroma and the knot was buried. A drop of moxifloxacin was placed in the eye and a shield was placed over it. Postoperatively, the patient was discharged with instructions to use moxifloxacin QiD.

The patient was seen in follow up 2 days later on 1/9/15 at 17:30. The patient complained of mild ocular irritation. Uncorrected visual acuity of the left eye was 20/25. Slit lamp examination showed clear cornea and anterior chamber. The fundus had a good red reflex. The patient was restarted on prednisolone OS QiD and nepafenac OS Qam and moxifloxacin OS QiD and told to follow up in 1 week. The patient called the office the next morning at 0700 complaining of severe vision loss and slight pain. The patient was seen immediately in the office at 0730. Eye examination showed VA of bare LP and IOP $15 \mathrm{mmhg}$. Slit lamp examination revealed an edematous cornea with a $1 \mathrm{~mm}$ hypopyon. There was no red-reflex. Exudate filled the entire posterior segment. The patient was immediately referred to retina and was seen 1 hour later. The vitreous was aspirated and sent for analysis. The cultures were negative. She was treated with Intravitreal vancomycin $(1 \mathrm{mg})$ and ceftazidime $(2 \mathrm{mg})$. Later that day she underwent a vitrectomy and anterior chamber wash out. Postoperatively she was placed on moxifloxacin QiD and prednisolone Q2hs. Her post-operative course showed gradual improvement. Her most recent eye examination was on 7/17/15 with no eye complaints. An eye examination show uncorrected VA of 20/30 OS, IOP was 10. Slit lamp exam was normal. Fundus exam was normal. The corneal suture was removed at six months without complications.

\section{Discussion}

Endophthalmitis is a clinical diagnosis and can be classified as either acute, subacute/delayed, or chronic. ${ }^{6}$ Characteristic findings on physical exam include injected conjunctiva, media haze, and a hypopyon in more than $80 \%$ of the cases. ${ }^{7,8}$ The diagnosis is supported by the culture of aqueous and vitreous samples which may be negative up to $30 \%$ of the time, as seen in our patient. (Table 1) lists the common bacterial organisms and the timeframe in which endophthalmitis usually occurs. ${ }^{9}$
Table I Classification of post-operative endophthalmitis

\begin{tabular}{ll}
\hline Classification of postoperative endophthalmitis \\
\hline & Gram Negative Bacteria \\
\hline Fulminant $<4$ days & $\begin{array}{l}\text { Streptococcus pneumonia } \\
\text { Staphylococcus aureus } \\
\text { Pseudomonas aeruginosa }\end{array}$ \\
Acute & Gram Negative Bacteria \\
2 -14 days & Staphylococcus aureus, \\
Mean 5-7 days & Staphylococcus epidermidis \\
Delayed onset/Subacute & Streptococcus species \\
2 weeks to months & fungi (Candida parapsilosis) \\
Mean 4-6 weeks & Corynebacterium sp. \\
& Propionibacterium acnes, \\
Chronic onset & Coagulase negative Staphylococcus \\
Months to years & Hemophilus influenza \\
Mean $>6$ weeks & Enterococci \\
& Staphylococcus species \\
\hline
\end{tabular}

This case is fascinating because of the delayed but fulminant presentation of endophthalmitis. Endophthalmitis was diagnosed 19 days post-op or 3.5 days after the patient was found to be seidel positive. The reopening of the wound may have been due to external trauma/friction from eye rubbing. Combined with a LASIK history, the patient's cornea may have been compromised and susceptible to disruption. Myopic LASIK severs collagen bands and thins the cornea, reducing its integrity and strength. ${ }^{10}$ The tensile strength of a cornea with a LASIK flap may be reduced significantly in comparison to a normal cornea. ${ }^{11}$ Although there are no documented cases of a patient with a history of LASIK developing a post cataract wound leak, we propose that these factors listed above may have demonstrated a significant role for the patient becoming seidel positive on day 15 .

However, in the OR the wound was found to be self-sealed and seidel negative. As a result of this finding, no intracameral antibiotics were given and a midstromal suture was placed. Evaluation 2.5 days later revealed excellent vision with minimal inflammation and irritation. However, 12 hours after that exam, the patient presented with a VA of bare light perception, intense intraocular pain, and an edematous cornea with a $1 \mathrm{~mm}$ hypopyon.

Our Initial clinical suspicion was a virulent streptococcus, staphylococcus aureus, or a pseudomonas bacteria due to the rapid progression, the large amount of vitreal exudate, and the dramatic loss of visual acuity. ${ }^{12-15}$ Due to poor vision, immediate pars plana vitrectomy and intravitreal vancomycin $1 \mathrm{mg}$ and ceftazidime $2 \mathrm{mg}$ were performed as recommend by EVS. ${ }^{9}$ However, patients with virulent forms of bacterial endophthalmitis including pseudomonas or alpha or beta-hemolytic streptococcus usually have poor visual outcomes regardless of quick treatment interventions. S. aureus is also associated with poor visual outcomes. More than $50 \%$ of reported cases resulted in final visual acuities of $20 / 400$ or worse. ${ }^{16,17}$ A few months after the incident, the patient had a VA of 20/30 OS and an unremarkable eye exam. However, in retrospect, other differentials and mechanisms have been considered. 
Another possible alternative to streptococcus and pseudomonas is an infection due to normal flora within the eye. The patient had a reopening of the sutured corneal incision and was thus exposed to normal bacterial flora. Also, improvement of visual acuity from bare LP to $20 / 30$ is much more common with immediate vitrectomy and intravitreal antibiotics in less virulent bacteria such as coagulase negative staph $^{9}$

However, another possible explanation is endophthalmitis involving suture placement or removal. Khurshid et al. ${ }^{18}$ documented 5 cases of suture induced endophthalmitis. In contrast to our patient, these patients presented over a period of 19 months with suture-related bacterial keratitis that progressed to endophthalmitis. No keratitis or inflammation was seen around the suture in our patient. Also, Culbert et al. ${ }^{19}$ documented 3 cases of endophthalmitis after suture removal. Loose sutures may serve as a nidus for bacterial growth causing inflammation and tissue breakdown, leading to endophthalmitis. ${ }^{18}$ One drop of ciprofloxacin was used before suture removal on postoperative day 15 . Although it may be possible, it is more probable the inflammation was due to an open corneal incision.

Another important differential to consider is a toxic, noninfectious insult such as toxic anterior segment syndrome. Toxic anterior segment syndrome is a sterile inflammatory reaction due to a noninfectious substance which enters the anterior segment. TASS may present similarly to endophthalmitis as both may present with decreased visual acuity, hypopyon, and fibrin accumulation. ${ }^{20}$ In contrast to TASS, which is mainly in the anterior chamber, our patient presented with intense posterior segment inflammation.

Since our patient was culture negative with a rapid progression of inflammation, TASS was an important differential to rule out before treatment. TASS has an early onset and may present as early as 12 to 48 hours postoperatively. ${ }^{21}$ The patient experienced symptoms three days after suture placement in the OR. Thus it is possible that the intense immune response may have been due to toxins or chemicals on the bandage contact lens or equipment used in the operating room. However, in the OR, the wound was found to be sealed and thus no intracameral injections were performed. TASS may occur due to abnormalities in the $\mathrm{pH}$ or ionic composition of irrigating saline solutions..$^{22}$ Nevertheless, BSS was only used topically. It is very unlikely that topical administration of BSS would lead to an intense inflammatory response.

Another possibility is delayed onset of TASS following cataract extraction. Cases have been documented with delayed onset inflammation ranging up to 21 days postoperative cataract surgery. Delayed onset TASS was associated with hydrophilic acrylic IOLs (memory lens). It was hypothesized that residual polishing compound on the memory lens was responsible for the postoperative inflammation. ${ }^{23}$ However, a memory lens was not used in this case but rather an Alcon SN60WF.

Delayed onset TASS has also been documented with entrance of ophthalmic ointment into the anterior segment post-surgery. Contamination with ophthalmic ointment was described as an oily material coating the anterior surface of the IOL, forming small globules within the anterior chamber. ${ }^{24}$ As a result, these patients developed a delayed TASS outside the typical window of 12 to 48 hours. However, there were no findings on slit lamp examination to support these findings. Overall, our patient showed no definitive signs of TASS including limbus-to-limbus corneal edema, high intraocular pressure, iris damage, and absence of vitritis. ${ }^{21}$ Thus we suggest an alternate hypothesis may be possible.
We suggest that fulminant culture negative endophthalmitis may be a variant of TASS in a patient who develops a good visual result. The patient was seidel positive for a period of 24 hours. It is possible that some non-infectious toxic stimulus entered the anterior chamber and made its way to the posterior segment, developing an intense inflammatory reaction that involved the posterior chamber as well. Below we devised a table weighing the pros versus cons of an endophthalmitis versus a variant of TASS (Toxic Posterior Segment Syndrome). Our culture and gram stain negative endophthalmitis may have been solved with a more sensitive diagnostic technique such as polymerase chain reaction known as PCR. PCR has been found to significantly aid in bacterial and fungal detection. ${ }^{25,26}$ In one real-time PCR study, detection of bacteria improved from $47.6 \%$ to $95.3 \%$. PCR results are also rapid, taking as little as 90 minutes to complete. However, the increased sensitivity may also be a drawback. PCR has a greater level of false positive results. Also, specimens need to be properly stored in order for PCR to be effective. ${ }^{26}$

The patient's improvement after vitrectomy and intravitreal antibiotics initially led us to a diagnosis of endophthalmitis even though a vitreous culture was negative. However, the excellent visual result in the face of bare LP, fulminant onset, combined with negative cultures makes the diagnosis unclear and possibly supports a variant of TASS, that we call Toxic posterior segment syndrome (TPSS).

\section{Conclusion}

Endophthalmitis is a rare complication of cataract surgery in the United States. ${ }^{1}$ Our patient presented with a delayed, fulminant endophthalmitis over the course of 3.5 days post seidel positive. Initial clinical suspicion was that the causative agent was a virulent bacteria organism due to the rapid progression and dramatic loss of visual acuity. However, the return of normal visual acuity post treatment with streptococcus, staphylococcus aureus, or pseudomonas endophthalmitis is unlikely. ${ }^{12-15}$ Nevertheless, the patient may have been an exception, developing a good visual outcome due to immediate therapeutic intervention. Intravitreal antibiotics were started an hour and a half after diagnosis with vitrectomy performed the same day, only a few hours later.

Endophthalmitis usually occurs within 1 to 2 weeks postoperatively. ${ }^{5}$ Endophthalmitis was diagnosed 19 days post CE or 3.5 days after the patient was found to be seidel positive. The reopening of the wound was hypothesized to have occurred from a weakened cornea from LASIK and presumed external friction while sleeping. We propose a controversial alternative hypothesis.Culturenegative fulminant endophthalmitis that has a good visual result post treatment may be due to a non-infectious toxic insult, a variant of TASS that we call Toxic Posterior Segment Syndrome (TPSS). The patient was seidel positive for a period of 24 hours. It is possible that some non-infectious toxic stimulus entered the anterior chamber and made its way to the posterior segment, developing an intense inflammatory reaction that involved the posterior segment as well. A syndrome we call Toxic Posterior Segment Syndrome. Factors that may support this ideology are listed in (Table 2).

In contrast, the severe anterior and posterior segment inflammation may have been due to bacteria, missed due to the lack of sensitivity of vitreal cultures. Thus, we recommend the incorporation of PCR as a diagnosing tool for endophthalmitis as the technology becomes more readily available for community centers. Although the rates of false positives are increased, sensitivity is significantly increased with a mean diagnosis achieved within 90minutes. 
Table 2 Clinical and biochemical variables of individuals with overweightobesity

\section{Endophthalmitis vs toxic posterior segment syndrome}

$\begin{array}{ll}\text { Support for Endophthalmitis } & \begin{array}{l}\text { Support for Toxic Posterior Segment } \\ \text { Syndrome }\end{array} \\ \begin{array}{l}\text { Fulminant <4 days post wound } \\ \text { opening dense vitreous infiltrate }\end{array} & \begin{array}{l}\text { Fulminant bacteria (Staph, strep, } \\ \text { and pseudomonas) do not have } \\ \text { good visual prognosis regardless of } \\ \text { treatment intervention and speed }\end{array} \\ \text { Seidel + for over } 24 \text { hours } & \begin{array}{l}\text { Seidel + for over 24 hours } \\ \text { Adnexa and fornices were irrigated }\end{array} \\ \text { No intracameral antibiotics given } & \begin{array}{l}\text { with 5\% povidone-iodine for preop } \\ \text { cataract surgery and moxifloxacin } \\ \text { QiD post-surgery until wound was } \\ \text { found open }\end{array}\end{array}$

SD, standard deviation; BMI, body mass index; WC, waist circumference; $A C$, abdominal circumference; HC, hip circumference; RER, respiratory exchange ratio; HR, hear rate

While PCR in a non-academic setting may be far off, another more practical prophylaxis may be the use of intracameral antibiotics in all patients. The Preferred Practice Pattern of the American Academy of Ophthalmology suggests there is mounting evidence showing intracameral antibiotics are highly efficacious in endophthalmitis prophylaxis. ${ }^{27} \mathrm{~A}$ retrospective analysis of more than 1.3 million patients revealed that intracameral antibiotics were associated with a 2.66-fold decrease of postoperative endophthalmitis compared to topical antibiotics. ${ }^{28}$ However, it is unclear what effect intracameral antibiotics would have had on our patient due to the unknown mechanism of pathology. Since intracameral antibiotics are compounded in the US it may have added to a cloudy differential of TASS or TPSS, especially if given after the postoperative wound leak. Although our patient presentation is highly atypical, our ASC has continued to use compounded intracameral cefuroxime for the past 1.5 years without complication.

\section{Acknowledgments}

None.

\section{Funding}

None.

\section{Conflicts of interest}

The authors declare there is no conflict of interests.

\section{References}

1. Lundstrom M, Wejde G, Stenevi U, et al. Endophthalmitis after cataract surgery: a nationwide prospective study evaluating incidence in relation to incision type and location. Ophthalmology. 2007;114(5):866-870.

2. Keay L, Gower EW, Cassard SD, et al. Postcataract surgery endophthalmitis in the United States: analysis of the complete2003 to 2004 Medicare database of cataract surgeries. Ophthalmology. 2012;119(5):914-922.

3. Aaberg TM, Flynn HW, Schiffman J, et al. Nosocomial acute-onset postoperative endophthalmitis survey: a 10-year review of incidence and outcomes. Ophthalmology. 1998;105(6):1004-1010.
4. Speaker MG, Milch FA, Shah MK, et al. Role of external bacterial flora in the pathogenesis of acute postoperative endophthalmitis. Ophthalmology. 1991;98(5):639-650.

5. Endophthalmitis Vitrectomy Study Group. Results of the Endophthalmitis Vitrectomy Study: a randomized trial of immediate vitrectomy and of intravenous antibiotics for the treatment of postoperative bacterial endophthalmitis. Arch Ophthalmol. 1995;113(12):1479-1496.

6. Safneck JR. Endophthalmitis: a review of recent trends. Saudi J Ophthalmol. 2012;26(2):181-189.

7. Lalwani GA, Flynn HW, Scott IU, et al. Acute-onset endophthalmitis after clear corneal cataract surgery (1996-2005). Clinical features, causative organisms, and visual acuity outcomes. Ophthalmology. 2008;115(3):473-476.

8. Taban M, Behrens A, Newcomb RL, et al. Acute endophthalmitis following cataract surgery: a systematic review of the literature. Arch Ophthalmol. 2005;123(5):613-620.

9. Endophthalmitis Vitrectomy Study Group. Results of the Endophthalmitis Vitrectomy Study. A randomized trial of immediate vitrectomy and of intravenous antibiotics for the treatment of postoperative bacterial endophthalmitis. Arch Ophthalmol. 1995;113(12):1479-1496.

10. Jaycock PD, Lobo L, Ibrahim J, et al. Interferometric technique to measure biomechanical changes in the cornea induced by refractive surgery. $J$ Cataract Refract Surg. 2005;31(1):175-184.

11. Schmack I, Dawson DG, McCarey BE, et al. Cohesive tensile strength of human LASIK wounds with histologic, ultrastructural, and clinical correlations. J Refract Surg. 2005;21(5):433-445.

12. Chen KJ, Sun MH, Lai CC, et al. Endophthalmitis caused by Pseudomonas aeruginosa in Taiwan. Retina. 2011;1(6):1193-1198.

13. Lee SY, Chee SP. Group B Streptococcus endogenous endophthalmitis: case reports and review of the literature. Ophthalmology. 2002;109(10):18791886 .

14. Miller JJ, Scott IU, Flynn HW, et al. Endophthalmitis caused by Streptococcus pneumoniae. Am J Ophthalmol. 2004;138(2):231-236.

15. Soriano F, Pérez-Trallero E, Pallarés R, et al. Spanish Pneumococcal Infection Study Network. Streptococcus pneumoniae endophthalmitis: a study of 36 cases with special reference to antibiotic resistance and treatment options. ClinMicrobiol Infect. 2006;12(6):519-526.

16. Shrader SK, Band JD, Lauter CB, et al. The clinical spectrum of endophthalmitis: incidence, predisposing factors, and features influencing outcome. J Infect Dis. 1990;162(1):115-120.

17. Callegan MC, Engelbert M, Parke DW, et al. Bacterial Endophthalmitis: Epidemiology, Therapeutics, and Bacterium-Host Interactions. Clin Microbiol Rev. 2002;15(1):111-124.

18. Khurshid GS, Fahy GT. Endophthalmitis secondary to corneal sutures: Series of delayed-onset keratitis requiring intravitreal antibiotics. $J$ Cataract Refract Surg. 2003;29(7):1370-1372.

19. Culbert RB, Devenyi RG. Bacterial endophthalmitis after suture removal. $J$ Cataract Refract Surg. 1999;25(5):725-727.

20. Bodnar Z, Clouser S, Mamalis N. Toxic anterior segment syndrome: Update on the most common causes. $J$ Cataract Refract Surg. 2012;38(11):1902-1910.

21. Mamalis N, Edelhauser HF, Dawson DG, et al. Toxic anterior segment syndrome. J Cataract Refract Surg. 2006;32(2):324-333.

22. Kreisler KR, Martin SS, Young CW, et al. Postoperative inflammation following cataract extraction caused by bacterial contamination of the cleaning bath detergent. $J$ Cataract Refract Surg. 1992;18(1):106-110. 
23. Jehan FS, Mamalis N, Spencer TS, et al. Postoperative sterile endophthalmitis (TASS) associated with the memorylens. $J$ Cataract Refract Surg. 2000;26(12):1773-1777.

24. Werner L, Sher JH, Taylor JR, et al. Toxic anterior segment syndrome and possible association with ointment in the anterior chamber following cataract surgery. J Cataract Refract Surg. 2006;32(2):227-235.

25. Sugita S, Kamoi K, Ogawa M, et al. Detection of Candida and Aspergillus species DNA using broad-range real-time PCR for fungal endophthalmitis. Graefes Arch Clin Exp Ophthalmol. 2012;250(3):391398.
26. Bispo PJ, de Melo GB, Hofling Lima AL, et al. Detection and Gram discrimination of bacterial pathogens from aqueous and vitreous humor using real-time PCR assays. Invest Ophthalmol Vis Sci. 2011;52(2):873881.

27. American Academy of Ophthalmology Cataract and Anterior Segment Panel. Preferred practice pattern guidelines. Cataract in the Adult Eye, San Francisco, CA: American Academy of Ophthalmology; 2011:19-20.

28. Javitt JC. Intracameral antibiotics reduce the risk of endophthalmitis after cataract surgery: does the preponderance of the evidence mandate a global change in practice?." Ophthalmology. 2016;123(2):226-231. 